\title{
A Switching Strategy for Target Tracking by Mobile Sensing Agents
}

\author{
Wencen $\mathrm{Wu}$ and Fumin Zhang \\ School of Electrical and Computer Engineering, Georgia Institute of Technology, Atlanta, USA \\ Email: \{wwencen3, fumin\}@gatech.edu
}

\begin{abstract}
We introduce a co-design approach for control and sensing algorithms for autonomous mobile sensing agents to track a moving target. The mobile sensing agents move in a group while taking bearings-only measurements. We construct an extended Kalman filter that provides estimates of the state of the target and investigate the optimal formation of the agent group by maximizing the determinant of Fisher information matrix (FIM). We develop a strategy that allows the agents to form groups with different number of agents. Agents autonomously switch to larger groups only when the convergence rate of smaller groups to the target is not satisfactory. This switching strategy strikes a balance between the complexity of cooperation and performance of sensing algorithms, which may enable more flexibility in autonomy compared to fixed strategies.
\end{abstract}

Index Terms - target tracking, bearings-only measurement, mobile sensing agents, switching strategy

\section{INTRODUCTION}

Target tracking is an important research area with a wide range of civilian and military applications [1]-[3]. By a mobile sensing agent, we refer to an unmanned autonomous vehicle (UAV) equipped with sensors that are able to detect a target. The sensors could be cameras, sonar, radars and thermal signature sensors, etc. Among all types of sensor observations, bearings-only target motion analysis (TMA) is a classical problem, in which an observer measures noisy bearings from a target and estimates the position and velocity of the target [4], [5]. With bearings-only observations, the measurement function is nonlinear. Various techniques have been developed to deal with the nonlinearity such as least squares, extended Kalman filter (EKF), maximum likelihood estimator, and particle filter.

When implementing the estimating techniques, observability issue arises when only one single sensing agent is used to track a target with bearings-only measurements. For the state of the target to be observable by a sensing agent, the agent is required to execute a proper maneuver to collect enough information, as described in [6], [7]. Recently, researchers have developed algorithms using sensor networks [8], [9] or multiple mobile sensing agents [1], [9]-[13] for target localization and tracking because measurements from multiple sensors can be fused and filtered to produce efficient and reliable state estimation.

Manuscript received June 13, 2012; accepted July 30, 2012

The research work is supported by ONR grants N00014-08-11007, N00014-09-1-1074, and N00014-10-10712 (YIP), and NSF grants ECCS-0841195 (CAREER), CNS-0931576, and ECCS-1056253.
Especially, if the number of sensing agents is limited and the agents have limited sensing range, mobile sensing agents are usually chosen to keep track of the target.

In this paper, we investigate the problem of using multiple mobile sensing agents to track one moving target. The objective is to estimate the target's position and velocity, and then control the mobile agents to follow the motion of the target to ensure the target is always detectable. To tackle this problem, one must solve an estimation problem and a control problem simultaneously. We use the constant velocity (CV) model for the target, and assume that the sensing agents take bearings-only measurements of the target. The state of the target, e.g. position, velocity, etc. can be estimated by filtering algorithms, which extracts the state information of the target from sensor measurements. Sensing agents will then be controlled to move towards the target while avoiding collisions between each other. The state estimation and motion planning have to be co-designed. For this purpose, we use the extended Kalman filter to produce state estimates and control the agents to move along the opposite direction of the gradient of a cost function, which depends on the norm of the relative distances between the the agent positions to the target position that is estimated by the extended Kalman filter. The motion of the agents aims to minimize the cost function. We also investigate the optimal formation of the agent group by maximizing the determinant of Fisher information matrix (FIM).

In typical scenarios, an agent group with more mobile sensing agents are expected to outperform an agent group with less agents or a static sensor network in terms of adaptability, scalability, and performance [14], [15]. However, target tracking with a large number of networked mobile agents increases communication delay and computational complexity. Because of the advantages and disadvantages of tracking with large groups and small groups, we believe that the tracking behavior of the sensing agents does not have to be fixed. Inspired by the switching behavior in fish groups [16] and the switching strategy developed in our previous papers regarding cooperative source seeking [17], we propose a switching strategy in this paper that allows the sensing agents to switch between groups with different number of agents. The switching conditions from smaller groups to larger groups are inspired and developed from the Razumikhin theorem [18]-[20]. Unlike the switching strategy introduced in [17], in which the switching conditions are applied to 
scalar fields, the switching conditions in this paper are considered in a field created by the cost function used for motion design. The switching strategy aims to allowing more flexible autonomy than fixed strategies.

The rest of the paper is organized as follows. First, we introduce the problem formulation of bearings-only target tracking. Next, we investigate optimal formations of the agent group and discuss the formation and motion control. We then propose the switching strategy that allows the agent group to switch between different group sizes. Simulation results are presented and conclusion remarks are provided in the last.

\section{PROBLEM FORMULATION}

Suppose a target is moving in a two dimensional plane with a constant velocity. Define $\mathbf{r}_{T}=\left(x_{T}, y_{T}\right)$ as the Cartesian coordinates of the target, and let $\dot{\mathbf{r}}_{T}=\left(\dot{x}_{T}, \dot{y}_{T}\right)$ represent the velocity of the target. $N$ sensing agents mounted with bearings-only sensors are used in tracking the target. Because of the limited number of sensing agents and the limited sensing range of each sensing agent, the sensing agents are controlled to move in a group to keep track of the target. Denote the position of the $i$ th agent as $\mathbf{r}_{i}=\left(x_{i}, y_{i}\right)$ and the corresponding bearing measurement as $\theta_{i}, i=1, \cdots, N$. Assume that the sensing agents move in a constant speed that is identical to the speed of the target. In addition, the following assumptions hold throughout the paper.

\section{A1. Each sensing agent obtains one measurement at} each time instant $k$.

A2. Each sensing agent is aware of the positions of all other agents.

Given the settings, at the $k$ th time instant, the geometry of the target tracking is illustrated in Figure 1, in which $\mathbf{r}_{i, k}$ represents the position of the $i$ th sensing agent, $\mathbf{r}_{T, k}$ represents the position of the target, and $\theta_{i, k}$ is the bearing from the $i$ th agent to the target. The objective of the target

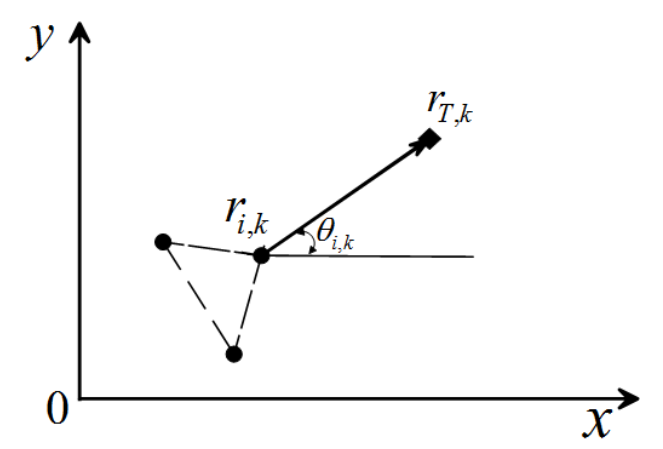

Figure 1. Bearings-only target tracking geometry in two-dimension.

tracking problem is to (1) estimate the state of the moving target, e.g. position and velocity from the bearings-only measurements taken by the $N$ sensing agents, and (2) control the sensing agents to keep track of of the target while remaining in a desired formation.
At each time instant, the bearing measurement of the $i$ th sensing agent is

$$
\theta_{i, k}=\arctan \frac{y_{T, k}-y_{i, k}}{x_{T, k}-x_{i, k}}+w_{i, k},
$$

where $w_{i, k} \sim \mathscr{N}\left(0, \sigma_{w}^{2}\right)$ is assumed to be mutually independent zero-mean Gaussian noise with variance $\sigma_{w}^{2}$. Choose the state of the target to be $\mathbf{s}=\left[x_{T}, y_{T}, \dot{x}_{T}, \dot{y}_{T}\right]$. Let $h\left(\mathbf{s}_{k}\right)$ be a $N \times 1$ observation matrix with the $i$ th row defined by $\theta_{i, k}$, that is,

$$
h\left(\mathbf{s}_{k}\right)=\left[\arctan \frac{y_{T, k}-y_{1, k}}{x_{T, k}-x_{1, k}}, \cdots, \arctan \frac{y_{T, k}-y_{N, k}}{x_{T, k}-x_{N, k}}\right]^{T},
$$

and let $\mathbf{y}_{k}=\operatorname{col}\left(\theta_{1, k}, \cdots, \theta_{N, k}\right) \in \mathscr{R}^{N}$ be the measurement vector consisting of all the measurements collected from the $N$ collaborating agents at time $k$. Then, the measurement equation for the $N$ sensing agents is

$$
\mathbf{y}_{k}=h\left(\mathbf{s}_{k}\right)+\mathbf{w}_{k} .
$$

The covariance of the noise vector $\mathbf{w}_{k}$ is given by $R=\sigma_{w}^{2} \mathbf{I}$, in which $\mathbf{I}$ is a $N \times N$ dimensional identity matrix. Therefore, the measurement vector $\mathbf{y}_{k}$ is a normally distributed random vector with mean $h\left(\mathbf{s}_{k}\right)$ and covariance matrix $R$, i.e., $\mathbf{y}_{k} \sim \mathscr{N}\left(h\left(\mathbf{s}_{k}\right), R\right)$.

The evolution of the target state is described by the dynamic model of a target. Most tracking algorithms are model based assuming the knowledge of the target motion is available. In this paper, we choose a constant-velocity model to describe the motion of the target, which is

$$
\mathbf{s}_{k+1}=F_{k} \mathbf{s}_{k}+\mathbf{v}_{k}
$$

where $F_{k}=\left(\begin{array}{cccc}1 & 0 & T & 0 \\ 0 & 1 & 0 & T \\ 0 & 0 & 1 & 0 \\ 0 & 0 & 0 & 1\end{array}\right)$ is the state transition matrix, $T$ is the sampling rate, $\mathbf{v}_{k} \sim \mathscr{N}(0, Q)$ is uncorrelated, zero-mean Gaussian noise processes with covariance matrices $Q$. The noise terms $\mathbf{v}_{k}$ and $\mathbf{w}_{k}$ satisfy:

$$
\begin{aligned}
E\left(\mathbf{v}_{k} \mathbf{v}_{j}^{T}\right) & =Q \delta_{k, j}, \\
E\left(\mathbf{w}_{k} \mathbf{w}_{j}^{T}\right) & =R \delta_{k, j}, \\
E\left(\mathbf{v}_{k} \mathbf{w}_{j}^{T}\right) & =0 .
\end{aligned}
$$

The uncertainty in the target state estimates will be influenced by the uncertainty of the bearing measurements and the positions of the sensing agents with respect to the target.

\section{EXTENDED KALMAN FILTER}

Since the measurement equation (3) is nonlinear, we apply the extended Kalman filter (EKF) to estimate the state of the target. Compared to particle filters, extended Kalman filter requires less computational load, which produces more efficient estimates.

Define $\mathbf{H}_{k}$ to be the Jacobian of the measurement vector with respect to the state of the target. We derive $\mathbf{H}_{k}=$ 
$\nabla_{\mathbf{s}} h\left(\mathbf{s}_{k}\right)=\left(\begin{array}{lll}\frac{\partial \theta_{1, k}}{\partial \mathbf{s}} & \cdots & \frac{\partial \theta_{N, k}}{\partial \mathbf{s}}\end{array}\right)^{T}$, the $i$ th row of which is

$\mathbf{H}_{i, k}=\left(-\frac{y_{T, k}-y_{i, k}}{\left(x_{T, k}-x_{i, k}\right)^{2}+\left(y_{T, k}-y_{i, k}\right)^{2}} \quad \frac{x_{T, k}-x_{i, k}}{\left(x_{T, k}-x_{i, k}\right)^{2}+\left(y_{T, k}-y_{i, k}\right)^{2}}\right.$

Two steps are consisted in the extended Kalman filter: prediction and update. Given state equation (4) and measurement equation (3), the equations of the EKF are listed below:

Prediction step:

$$
\begin{aligned}
\hat{\mathbf{s}}_{k}^{-} & =F_{k} \hat{\mathbf{s}}_{k-1}, \\
P_{k}^{-} & =F_{k} P_{k-1} F_{k}^{T}+Q_{k-1}, \\
K_{k} & =P_{k}^{-} \mathbf{H}_{k \mid \hat{\mathbf{s}}_{k}^{-}}^{T}\left(\mathbf{H}_{k \mid \hat{\mathbf{s}}_{k}^{-}} P_{k}^{-} \mathbf{H}_{k \mid \hat{\mathbf{s}}_{k}^{-}}^{T}+R_{k}\right)^{-1},
\end{aligned}
$$

where $P_{k}$ is the error covariance matrix of the extended Kalman filter, and $K_{k}$ is the Kalman gain.

Update step:

$$
\begin{aligned}
P_{k} & =\left(I-K_{k} \mathbf{H}_{k \mid \hat{\mathbf{s}}_{k}^{-}}\right) P_{k}^{-}, \\
\hat{\mathbf{s}}_{k} & =\hat{\mathbf{s}}_{k}^{-}+K_{k}\left(\mathbf{y}_{k}-h\left(\hat{\mathbf{s}}_{k}^{-}\right)\right) .
\end{aligned}
$$

Consider the linearized system

$$
\begin{aligned}
\mathbf{s}_{k+1} & =F_{k} \mathbf{s}_{k}+\mathbf{v}_{k}, \\
\mathbf{y}_{k} & =\mathbf{H}_{k} \mathbf{s}_{k}+\mathbf{w}_{k} .
\end{aligned}
$$

If only one agent is used in tracking the target, $N=1$. Then, the observation matrix becomes $\mathbf{H}_{k}=$ $\left(\begin{array}{llll}-\frac{y_{T, k}-y_{1, k}}{\left(x_{T, k}-x_{1, k}\right)^{2}+\left(y_{T, k}-y_{i, k}\right)^{2}} & \frac{x_{T, k}-x_{1, k}}{\left(x_{T, k}-x_{1, k}\right)^{2}+\left(y_{T, k}-y_{i, k}\right)^{2}} & 0 & 0\end{array}\right)$, which implies that the system is unobservable. In fact, for the state of a moving target to be observable to a sensing agent, the sensing agent must execute a proper maneuver, e.g. changes the heading or accelerates, as described in [6], [7]. Since we assume that the speed of the sensing agents are the same of the target, we always let $N \geq 2$ in this paper.

\section{FORMATION AND MOTION CONTROL}

For the control problem, we design formation control laws that control the $N$ sensing agents to remain in a desired formation and motion control laws that control the entire formation to move towards the target. The desired formation aims to minimize the error variance in estimating the state of the target. In this section, we first introduce the optimal formation that the agent group is required to maintain, then, we discuss the formation control and motion control design for the group.

\section{A. Optimal Formation}

The Fisher information matrix (FIM) describes the amount of information that the measurement $\mathbf{y}_{k}$ carries about the unobservable state $\mathbf{s}_{k}$. It is calculated in [21] that the FIM is

$$
I\left(\mathbf{s}_{k}\right)=\nabla_{\mathbf{s}} h\left(\mathbf{s}_{k}\right)^{T} R^{-1} \nabla_{\mathbf{s}} h\left(\mathbf{s}_{k}\right) .
$$

Recall the error covariance matrix $P_{k}$ in the extended Kalman filter, which can also be written as

$$
P_{k}^{-1}=\left(P_{k}^{-}\right)^{-1}+\mathbf{H}_{k \mid \hat{\mathbf{s}}_{k}^{-}}^{T} R^{-1} \mathbf{H}_{k \mid \hat{\mathbf{s}}_{k}^{-}} .
$$

The second term of $P_{k}^{-1}$ can be recognized as the Fisher information matrix since

$0) I\left(\hat{\mathbf{s}}_{k}^{-}\right)=\nabla_{\mathbf{s}} h\left(\hat{\mathbf{s}}_{k}^{-}\right)^{T} R^{-1} \nabla_{\mathbf{s}} h\left(\hat{\mathbf{s}}_{k}^{-}\right)=\mathbf{H}_{k \mid \hat{\mathbf{s}}_{k}^{-}}^{T} R^{-1} \mathbf{H}_{k \mid \hat{\mathbf{s}}_{k}^{-}}$.

Therefore, equation (12) becomes

$$
P_{k}^{-1}=\left(P_{k}^{-}\right)^{-1}+I\left(\hat{\mathbf{s}}_{k}^{-}\right) .
$$

Hence, reducing the estimating error in the extended Kalman filter can be translated into increasing the Fisher information. The Cramer-Rao lower bound (CRLB) states that the variance of any unbiased estimator is bounded by the inverse of the Fisher information. As stated in [21][24], an agent configuration over the space of all angle positions $\theta_{i}, \forall i \in\{1, \cdots, N\}$ is optimal if the configuration maximizes the determinant of Fisher information matrix, or minimizes the Cramer-Rao lower bound.

Define the distance from the target to the $i$ th sensing agent as $d_{i}=\left\|\mathbf{r}_{T}-\mathbf{r}_{i}\right\|$. We derive that

$$
\begin{aligned}
I(\mathbf{s}) & =\frac{1}{\sigma_{w}^{2}}\left(\begin{array}{cccc}
\sum_{i=1}^{N} \frac{1}{d_{i}^{2}} \sin ^{2} \theta_{i} & -\sum_{i=1}^{N} \frac{1}{2 d_{i}^{2}} \sin 2 \theta_{i} & 0 & 0 \\
-\sum_{i=1}^{N} \frac{1}{2 d_{i}^{2}} \sin 2 \theta_{i} & \sum_{i=1}^{N} \frac{1}{d_{i}^{2}} \cos ^{2} \theta_{i} & 0 & 0 \\
0 & 0 & 0 & 0 \\
0 & 0 & 0 & 0
\end{array}\right) \\
& =\frac{1}{\sigma_{w}^{2}}\left(\begin{array}{cc}
I^{\prime}(\mathbf{s}) & \mathbf{0} \\
\mathbf{0} & \mathbf{0}
\end{array}\right),
\end{aligned}
$$

in which $\mathbf{0}$ is a $2 \times 2$ matrix. If we only consider the Fisher information of the position measurements, that is, $I^{\prime}(\mathbf{s})$, then, the optimal formation can be obtained by solving the following two equations simultaneously [21]-[24]

$$
\sum_{i=1}^{N} \frac{1}{d_{i}^{2}} \sin 2 \theta_{i}=0, \text { and } \sum_{i=1}^{N} \frac{1}{d_{i}^{2}} \cos 2 \theta_{i}=0 .
$$

In addition, $\theta_{i}$ can be found if and only if $\frac{1}{d_{j}^{2}} \leq \sum_{i=1, i \neq j}^{N} \frac{1}{d_{i}^{2}}$ for all $j \in\{1, \cdots, N\}$. It is also proved in [24] that if there are only indices permutation, agents flipping about the target, or global rotation, reflection of combined, the two placements $\left\{\theta_{i}\right\}_{i=1}^{N}$ and $\left\{\theta_{i}^{\prime}\right\}_{i=1}^{N}$ are equivalent.

In [21]-[24], the target that is being localized by sensors is static, and $d_{i}$ are considered as the sensor ranges, which are fixed. If the sensor ranges are identical, i.e., $d_{i}=d_{j}, \forall i, j \in\{1, \cdots, N\}, i \neq j$, the angles between adjacent sensing agents can be calculated as $\theta_{i, j}=\frac{2 \pi}{N}$. Figure 2 (a) illustrates an example of an optimal formation of three sensing agents with identical distances $d_{i}$ from the target. In this case, $\theta_{1,2}=\theta_{2,3}=\theta_{3,1}=\frac{2 \pi}{3}$.

In this paper, the sensing agents are tracking a moving target, and may not start from locations close to the target. Therefore, the agents may not be controlled to be placed around the target. We solve this problem by flipping sensor agents about the target so that all the agents are in the same side with regard to the target. In addition, we do not assume that each sensing agent has fixed sensing range. We assume that the sensing agents can take bearing measurements regardless of the distances from the target, which indicates that $d_{i}$ may change before the distances between the sensing agents and the target converge. Thus, 
the optimal formation evolves with the change of the distances $d_{i}$.

Figure 2 (b) illustrates an optimal formation of three sensing agents by flipping the second agent about the target in Figure 2 (a). As shown in the figure, the dashed lines connecting three sensing agents form a triangular formation. Note that the calculation of the optimal formation only determines $\theta_{i}$, which indicates that the formation size in terms of the distance between agents depends on $d_{i}$. Then, we can observe from Figure 2 (b) that, as the distances from agents to the target decrease, i.e., decrease $d_{i}$, the formation size in terms of the distances between agents decreases.

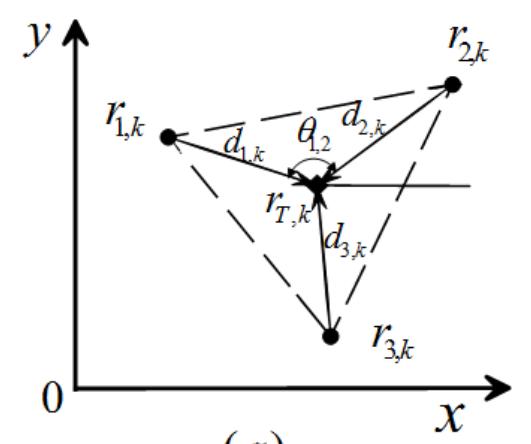

(a)

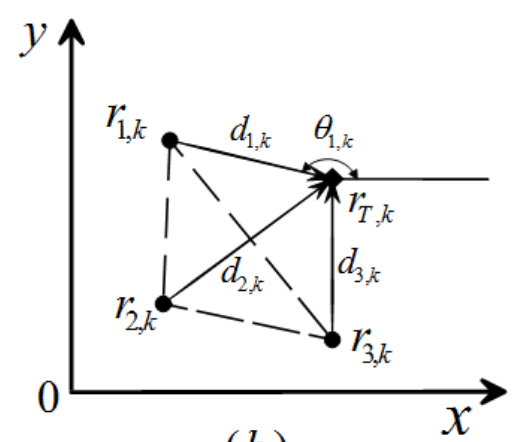

(b)

Figure 2. Bearings-only target tracking geometry in two-dimension.

\section{B. Formation Control}

For $N$ sensing agents tracking a target, the agents are controlled to maintain a desired formation, as described in the previous section, and the entire formation moves towards the target. Denote the center of the formation as $\mathbf{r}_{c, k}=\frac{1}{N} \sum_{i=1}^{N} \mathbf{r}_{i, k}$. The formation shape is described using Jacobi vectors that satisfy $\left[\mathbf{r}_{c, k}, \mathbf{q}_{1, k}, \cdots, \mathbf{q}_{N-1, k}\right]=$ $\left[\mathbf{r}_{1, k}, \mathbf{r}_{2, k}, \cdots, \mathbf{r}_{N, k}\right] \Psi$, in which $\mathbf{q}_{i, k}$ are Jacobi vectors and $\Psi$ is the Jacobi transform. For example, if we deploy three agents, the Jacobi vectors are

$$
\begin{aligned}
& \mathbf{q}_{1, k}=\frac{\sqrt{2}}{2}\left(\mathbf{r}_{2, k}-\mathbf{r}_{3, k}\right) \\
& \mathbf{q}_{2, k}=\frac{\sqrt{6}}{6}\left(2 \mathbf{r}_{1, k}-\mathbf{r}_{2, k}-\mathbf{r}_{3, k}\right),
\end{aligned}
$$
and the Jacobi transform $\Psi=\left(\begin{array}{ccc}\frac{1}{3} & \frac{1}{3} & \frac{1}{3} \\ 0 & \frac{\sqrt{2}}{2} & -\frac{\sqrt{2}}{2} \\ \frac{\sqrt{6}}{3} & -\frac{\sqrt{6}}{6} & -\frac{\sqrt{6}}{6}\end{array}\right)$.
The Jacobi transform decouples the kinetic
energy of the entire system [25], [26], which enables us to design separate control laws for the formation center motion and the formation shape.

We assume Jacobi vectors $\mathbf{q}_{j, k}$ obey second order dynamics $\ddot{\mathbf{q}}_{j, k}=u_{j, k}$, in which $u_{j, k}$ are forces applied to $\mathbf{q}_{j, k}$. Then, the control laws can be designed as

$$
u_{j, k}=-K_{1}\left(\mathbf{q}_{j, k}-\mathbf{q}_{j, k}^{0}\right)-K_{2} \dot{\mathbf{q}}_{j, k}, j=1, \cdots, N-1,
$$

where $K_{1}$ and $K_{2}$ are positive constant gains and $\mathbf{q}_{j, k}^{0}$ are desired vectors that define a formation. $\mathbf{q}_{j, k}^{0}$ can be determined by the optimal formation calculated according to the previous section. The control laws have an exponential rate of convergence. $\mathbf{q}_{i, k}$ can be calculated by integrating the control forces $u_{j, k}$ twice.

After obtaining the position of the formation center $\mathbf{r}_{c, k+1}$ using motion control laws and Jacobi vectors $\mathbf{q}_{j, k}$ at step $k$, we can take the inverse Jacobi transform to get the new positions of the agents $\mathbf{r}_{i, k+1}, i=1, \cdots, N$ by

$\left[\mathbf{r}_{1, k+1}, \mathbf{r}_{2, k+1}, \cdots, \mathbf{r}_{N, k+1}\right]=\left[\mathbf{r}_{c, k+1}, \mathbf{q}_{1, k+1}, \cdots, \mathbf{q}_{N-1, k+1}\right] \Psi^{-T}$.

In this way, the positions of the agents at each step are determined, and the formation of the agents are maintained.

\section{Group Motion Planning}

Define a simple convex quadratic cost function

$$
V_{c, k}=\frac{1}{2}\left(\mathbf{r}_{c, k}-\mathbf{r}_{T, k}\right)^{T} M\left(\mathbf{r}_{c, k}-\mathbf{r}_{T, k}\right),
$$

where $M$ is a symmetric matrix. Equation (20) yields

$$
\nabla V_{c, k}=M\left(\mathbf{r}_{c, k}-\mathbf{r}_{T, k}\right) .
$$

Let the agent group move towards the target along the gradient of $V_{c, k}$.

$$
\mathbf{r}_{c, k+1}=\mathbf{r}_{c, k}-\frac{\nabla V_{c, k}}{\left|\nabla V_{c, k}\right|} v=\mathbf{r}_{c, k}-\frac{\mathbf{r}_{c, k}-\mathbf{r}_{T, k}}{\left|\mathbf{r}_{c, k}-\mathbf{r}_{T, k}\right|} v,
$$

where $v$ is the speed of the agent, which is assumed to be constant. The agent moves in the direction that reduces the value of the cost function until it gets sufficient close to the target.

\section{THE SWITCHING STRATEGY}

As we discussed in the extended Kalman filter section, the number of agents in a group should be greater or equal to two so that the observability of the system can be ensured. However, if we just use two agents, there are situations that the noise strength is high so that a group with two agent may not be able to keep track of the target. In these cases, a group with more agents are required to produce state estimates of the target. With the increased number of the agents in a group, the complexity of cooperation increases. Therefore, we propose a switching 
strategy in this section that balances the tradeoffs of the performance and cost between the smaller groups and larger groups in terms of the number of agents.

Suppose we deploy even number of agents and first let the agents form groups with two agents. The center of the $i$ th two-agent group is denoted by $\mathbf{r}_{c, k}^{i}$, in which $i=1, \cdots, \frac{N}{2}$. We always treat agent-groups as "superagents" since we can design separate control laws for the formation control and formation center motion control.

We assume that each mobile sensing agent has a finite memory length to store information (positions, velocities, values of cost function, etc) gathered over time. For an agent group, the memory length of the group can be considered as either the weighted average or the sum of the memory lengths of all the agents in the group. In this paper, we assume that the memory lengths of each agent and each agent group are identical.

In the tracking process, the $N$ agents first form $\frac{N}{2}$ two-agent groups. For each group, a cost function $V_{c, k}^{i}$ in Equation (20) is calculated at each time instant. The two-agent groups keep tracking the target using the state estimates from the extended Kalman filter and the formation and motion control laws derived in previous sections until they are not guaranteed to move towards the target at a convergence rate that is compatible with their memory lengths. To examine if a convergence rate is satisfactory, we introduce sufficient conditions for a group to converge to a constant distance from the target position. The sufficient conditions then serve as switching conditions that decide if the agent group needs to switch from smaller groups to larger groups. If the sufficient conditions are satisfied, then a satisfactory convergence rate will not be guaranteed. The agents will start forming larger groups.

The switching conditions are based on the Razumikhin theorem, which was originally developed for verifying the stability of time-delay systems [18]-[20]. We first restate the Razumikhin theorem for the asymptotic stability of time-delay systems [20] without proof.

Theorem 1(Razumikhin) Given a system $\dot{x}(t)=f\left(t, x_{t}\right)$ where $x \in \mathbb{R}^{n}$ and $x_{t} \in \mathscr{C}$ represents the delayed system trajectory, suppose $f: \mathbb{R} \times \mathscr{C} \rightarrow \mathbb{R}^{n}$ takes bounded subsets of $\mathscr{C}$ into bounded subsets of $\mathbb{R}^{n}$. Suppose $\alpha_{1}, \alpha_{2}, w: \mathbb{R}_{+} \rightarrow \mathbb{R}_{+}$are continuous nondecreasing functions, $\alpha_{1}(u)>0, \alpha_{2}(u)>0$ and $w(u)>0$ for $u>0$, and $\alpha_{1}(0)=\alpha_{2}(0)=0, \alpha_{2}$ strictly increasing. Suppose there exists a continuous nondecreasing function $g(u)>u$ for $u>0$. If there exists a continuous differentiable function $V: \mathbb{R} \times \mathbb{R}^{n} \rightarrow \mathbb{R}$ such that

$$
\alpha_{1}(\|x\|) \leq V(t, x) \leq \alpha_{2}(\|x\|), \forall t \in \mathbb{R}, x \in \mathbb{R}^{n},
$$

and the derivative of $V$ along the solution $x(t)$ satisfies

$$
\dot{V}(t, x(t)) \leq-w(\|x(t)\|)
$$

whenever $V((t+\theta), x(t+\theta)) \leq g(V(t, x(t)))$ for all $\theta \in$ $[-r, 0]$, then the equilibrium $x(t)=0$ of the system is asymptotically stable.
For discrete systems, condition (24) becomes [18]

$$
V(k+1, x(k+1))-V(k, x(k)) \leq-w(\|x(k)\|),
$$

whenever $V((k+\theta), x(k+\theta)) \leq g(V(k, x(k)))$ for all $\theta \in$ $[-r, 0]$.

At each time step $k$, the cost function $V_{c, k}^{i}$ is calculated by each agent group. For simplicity, we drop the subscript $i$ used to index the group in the following arguments. Suppose that each agent has a memory with finite length $r$, in which $r \in z^{+}$. The memory is used to store the previous values of the cost function $V_{c, k+s}$, in which $s$ is a nonpositive integer such that $-r \leq s \leq 0$. Then, based on the discrete time Razumikhin theorem, we have the following proposition.

Proposition 1 Let $\bar{V}_{c, k}=\max _{s \in[-r, 0]} V_{c, k+s}$ where $r \in z^{+}$. Suppose $V_{\min } \leq V_{c, k} \leq V_{\max }$, in which $V_{\min } \geq 0$ depending on the initial position of $\mathbf{r}_{c, k}$. If $V_{c, k+1}-V_{c, k} \leq K_{1} V_{c, k}$ whenever $\bar{V}_{c, k} \leq K_{2} V_{c, k}$, in which $K_{1}, K_{2}>0$ are constants, then $V_{c, k}$ will converge to $V_{\min }$ as $k \rightarrow \infty$, which means the distance between $\mathbf{r}_{c, k}$ and $\mathbf{r}_{T, k}$ will converge to a constant.

The proof for the proposition is straight-forward from the proof of the Razumikhin theorem, so we omit it here. Given the above proposition, we propose the following tracking algorithm for target tracking.

Algorithm 1 Suppose $N$ sensing agents with memory length $r$ are tracking a moving target. $N$ is an even number. The $N$ agents form $\frac{N}{2}$ two-agent groups at step $k=0$. At step $k$, do the following:

$\mathrm{S} 1$. Each agent takes a bearings-only measurement $y_{i, k}$ of the target;

S2. Each two-agent group obtains the state estimation produced by the extended Kalman filter;

S3. Given the state estimation, each two-agent group calculates the value of the cost function $V_{c, k}$ (20) and moves towards the target according to equation (22); If $k=T_{\text {terminal }}$, in which $T_{\text {terminal }}$ is the terminal time, stop.

S4. If $k \leq r$, go to step S1. Otherwise, continue to step S5.

S5. If $\bar{V}_{c, k} \leq K_{2} V_{c, k}$, go to step $\mathrm{S} 1$. Otherwise, continue to step S6;

S6. The agent group moves one step further, and obtain a new cost function value $V_{c, k+1}$. Then, the group checks the value of $V_{c, k+1}-V_{c, k}$. If $V_{c, k+1}-V_{c, k} \leq$ $K_{1} V_{c, k}$, then, it remains in the two-agent group. Go to step S1. Otherwise, continue to step S7;

S7. The group requires to switch to larger groups with more agents by sending switching signals to other groups.

Under the above algorithm, if for all $k>0, V_{c, k+1}-$ $V_{c, k} \leq K_{1} V_{c, k}$ whenever $\bar{V}_{c, k} \leq K_{2} V_{c, k}$, the distance between the agent group and the target will converge to a constant according to Proposition 1. According to Proposition 1 and Algorithm 1, the switching conditions from smaller groups to larger groups can be stated as: (1) $\bar{V}_{c, k} \leq K_{2} V_{c, k}$, and (2) $V_{c, k+1}-V_{c, k} \leq K_{1} V_{c, k}$. Once a two-agent group detects that both switching conditions are satisfied, it notifies other two-agent groups, then all two-agent groups 
switch to cooperative tracking upon request. All the agents form a $\mathrm{N}$-agent group with similar formation control laws and motion control laws developed in previous sections. This ensures that all agents behave consistently in the cooperative tracking mode.

One drawback of the proposed algorithm is that when one two-agent group initiates cooperation, all the twoagent groups will need to cooperate. As we can tell from the extended Kalman filter, when the number of collaborating agents increases, the estimation error of the state of the target decreases. However, due to the increased cost associated with collaboration, it may not be efficient to have all agent groups collaborating with each other. Therefore, we modify the algorithm to allow the agent groups to gradually form larger groups.

To state the modified algorithm, let's first review the definition of the nearest neighbor to an agent group $i$. Let $d_{i, j}=\left|\mathbf{r}_{c, k}^{i}-\mathbf{r}_{c, k}^{j}\right|$ be the distance between agent group $i$ and $j, i, j \in\left(1, \cdots, \frac{N}{2}\right), i \neq j$. We call an agent group $n$ the nearest neighbor to agent $i$ if $\min _{j \in\left(1, \frac{N}{2}\right), j \neq i} d_{i, j}=d_{i, n}$. Then, after step S6 of Algorithm 1, only the nearest neighbor to the agent group that requires collaboration joins the group and form a group with $N=4$ agents. The four-agent group checks the switching conditions at each time step while moving as a "super-agent" to track the target. Only when the four-agent group fails to track the target with a satisfactory convergence rate determined by the switching conditions, it requires more agent groups to join it and form larger groups. In this way, the agents gradually form larger groups from smaller groups. This strategy guarantees the convergence without requiring all agents to collaborate, which allows more flexibility of balancing the cost and performance of using different number of agents in a group.

The difference between the switching strategy introduced in [17] and the one discussed in this paper is that the switching conditions in this paper are evaluated in a cost function, which is calculated at every step based on the state estimates from the extended Kalman filter, instead of in a scalar field, which is directly measured by the sensing agents. Therefore, the switching strategy can be generalized to other applications as long as a cost function is defined. In addition, note that the switching strategy is valid when the speed of the target and the sensing agents are identical. If the speed of the sensing agents is less than the speed of the target, the sensing agents will lost the track of the target. If the speed of the sensing agents is greater than that of the target, the positions of the sensing agents will converge to the position of the target.

\section{SIMULATION RESULTS}

We simulate a group of agents tracking a target moving in constant velocities. In the simulations, we choose $T=$ $0.1, \sigma_{w}=0.4$, and $\sigma_{v}=0.2$. At each time step, the sensing agents take bearings-only measurements from the target. A central controller collects the measurements and runs an extended Kalman filter that produces state estimates, which are used in calculating the motion control laws and formation control laws. We obtain optimal formations according to Equation (16). In the case of two-agent groups, the state of the target is unobservable if the target is located on the line that connects the two sensing agents. Therefore, we choose another form of optimal formation that the two lines connecting each agent and the target are perpendicular. Figures 3 and 4 illustrate the tracking results of a two-agent group and a fouragent group, respectively. In the figures, we use different colored dots to represent the positions of the agents and plot the agent formations every 50 steps. The red lines are the trajectories of the moving target, and the black lines are the trajectories of the center of the groups. As illustrated in the figures, as the formation moves closer to the target, the size of the optimal formation reduces. The green dots illustrate the estimated positions of the moving target produced by the extended Kalman filter. The green dots in the two figures indicate that the error in the estimates is reduced by increasing the number of agents in a group.

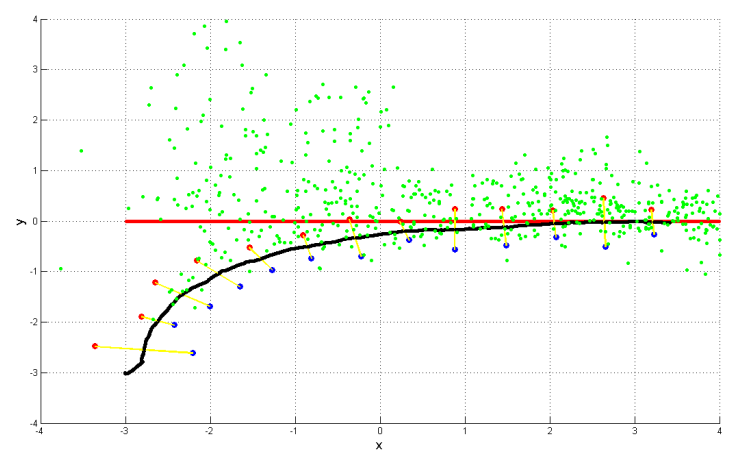

Figure 3. The trajectory of a two-agent group tracking a moving target.

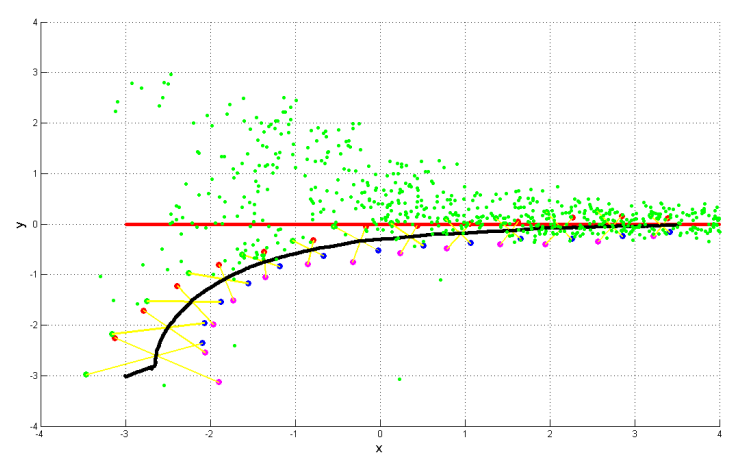

Figure 4. The trajectory of a four-agent group tracking a moving target.

We then implement the switching strategy using four sensing agents. As illustrated in Figure 5, we first let the four agents form two two-agent groups and track the moving target using the techniques introduced previously. The blue and cyan dots are trajectories of the two twoagent groups, and the magenta and yellow dots are the 
estimated positions of the target by the two two-agent groups, respectively. The two-agent groups check the switching conditions at each time step while moving towards the target based on the estimated target positions. Once the switching conditions are satisfied, which indicates that the convergence to the constant distance from the target is not guaranteed, the two two-agent groups join together and form a four-agent group. In this simulation, the switching occurs at step $k=65$. The black dots are the trajectories of the four-agent group, and the green dots are the estimated positions of the target. Figure 6 shows the estimated speed by the four-agent group. The red line is the real speed of the target, which is 0.1 . Figure 7 shows the relative distance between the center of the fouragent group and the moving target, which indicates that the relative distance converges.

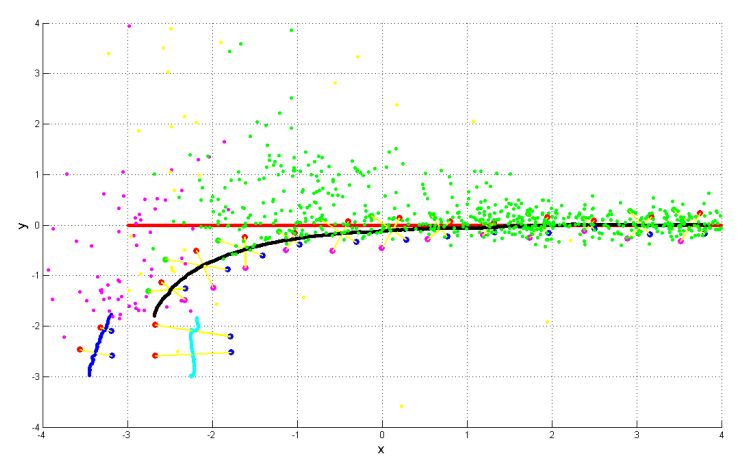

Figure 5. The trajectories of the agent groups and the estimates in the switching strategy.

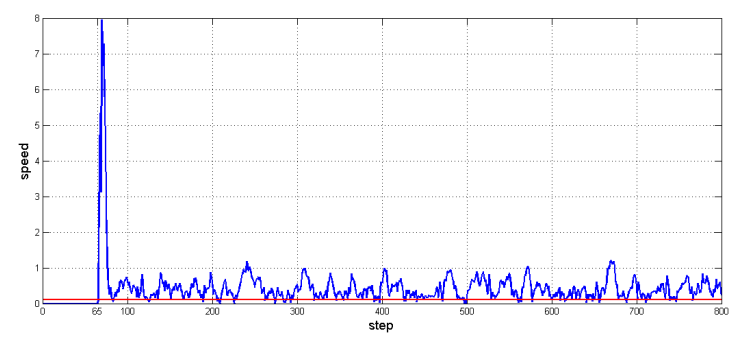

Figure 6. The estimated speed of the target after switching to a fouragent group.

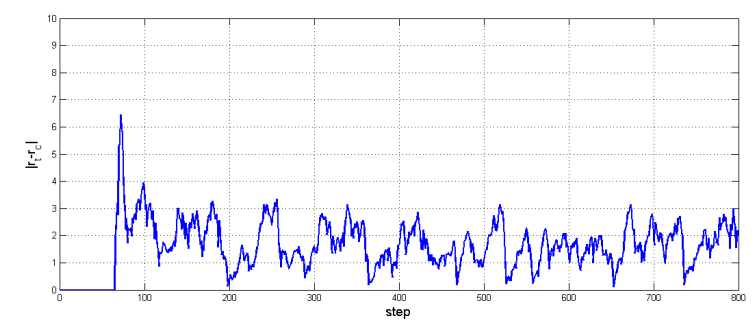

Figure 7. The relative distance between the four-agent group and the target.

\section{CONCLUSIONS}

In this paper, we propose a switching strategy for target tracking using mobile sensing agents that take bearingsonly measurements. The switching strategy allows the agents to switch between different group sizes, which balances the complexity of cooperation and performance of sensing algorithms and allows more flexibility in tracking targets than fixed strategies.

\section{REFERENCES}

[1] M. Wheeler, B. Schrick, W. Whitacre, M. Campbell, R. Rysdyk, and R. Wise, "Cooperative tracking of moving targets by a team of autonomous UAVs," in Proc. IEEE/AIAA 25th Digital Avionics Systems Conf, 2006, pp. $1-9$.

[2] P. Zhan, D. W. Casbeer, and A. L. Swindlehurst, "A centralized control algorithm for target tracking with UAVs," in Proc. Conf Signals, Systems and Computers Record of the Thirty-Ninth Asilomar Conf, 2005, pp. 1148-1152.

[3] F. Zhang, D. M. Fratantoni, D. Paley, J. Lund, and N. E. Leonard, "Control of coordinated patterns for ocean sampling," International Journal of Control, vol. 80, pp. 11861199, 2007.

[4] A. Farina, "Target tracking with bearings-only measurements," Signal Processing, vol. 78, no. 1, pp. 61-78, 1999.

[5] K. Dogancay, "Bearings only target localization using total least squares," Signal Processing, vol. 85, no. 9, pp. 16951710, 2003.

[6] S. Nardone and V. J. Aidala, "Observability criteria for bearings-only target motion analysis," IEEE Transactions on Aerospace and Electronic Systems, vol. 17, no. 2, pp. 162-166, 1981.

[7] T. L. Song, "Observability of target tracking with bearingsonly measurements," IEEE Transactions on Aerospace and Electronic Systems, vol. 32, no. 4, pp. 1468-1472, 1996.

[8] R. R. Brooks, P. Ramanathan, and A. M. Sayeed, "Distributed target classification and tracking in sensor networks," Proceedings of the IEEE, vol. 91, no. 8, pp. 11631171, 2003.

[9] S. Oh, L. Schenato, P. Chen, and S. Sastry, "Tracking and coordination of multiple agents using sensor networks: System design, algorithms and experiments," Proceedings of the IEEE, vol. 95, no. 1, pp. 234-254, 2007.

[10] Z. Tang and U. Ozguner, "Motion planning for multitarget surveillance with mobile sensor agents," IEEE Transactions on Robotics, vol. 21, no. 5, pp. 898-908, 2005.

[11] B. Bethke, M. Valenti, and J. How, Advances in Cooperative Control and Optimization, 2007, vol. 369, ch. Cooperative Vision Based Estimation and Tracking Using Multiple UAVs, pp. 179-189.

[12] M. E. Campbell and W. W. Whitacre, "Cooperative tracking using vision measurements on seascan UAVs," IEEE Transactions on Control Systems Technology, vol. 15, no. 4, pp. 613-626, 2007.

[13] R. Olfati-Saber, "Distributed tracking for mobile sensor networks with information-driven mobility," in Proc. American Control Conf. ACC '07, 2007, pp. 4606-4612.

[14] Y. Cao, A. Fukunaga, and A. Khang, "Cooperative mobile robotics: Antecedents and directions," Autonomous Robots, vol. 4, no. 1, pp. 7-27, 1997.

[15] G. Dudek, M. Jenkin, E. Milios, and D. Wilkes, "A taxonomy for multiagent robotics," Autonomous Robots, vol. 3, no. 4, pp. 375-397, 1996.

[16] C. Torney, Z. Neufeld, and L. Couzin, "Context-dependent interaction leads to emergent search behavior in social aggregates," Proc. of the National Academy of Sciences, vol. 106, no. 52, pp. 22 055-22 060, 2009. 
[17] W. Wu and F. Zhang, "Robust cooperative exploration with a switching strategy," IEEE Transactions on Robotics, vol. 28, no. 4, pp. 828-839, 2012.

[18] S. Elaydi and S. Zhang, "Stability and periodicity of difference equations with finite delay," Funkcialaj Ekvacioj, vol. 37, pp. 401-413, 1994.

[19] S. Zhang and M. Chen, "A new Razumikhin theorem for delay difference equations," Computers \& Mathematics with Applications, vol. 36, pp. 10-12, 1998.

[20] K. Gu, V. L. Kharitonov, and J. Chen, Stability of TimeDelay Systems. Boston: Birkhäuser, 2003.

[21] S. Martnez and F. Bullo, "Optimal sensor placement and motion coordination for target tracking," Automatica, vol. 42, no. 4, pp. 661-668, 2006.

[22] K. Doanay and H. Hmam, "Optimal angular sensor separation for AOA localization," Signal Processing, vol. 88, no. 5, pp. 1248-1260, 2008.

[23] A. N. Bishop, B. Fidan, B. D. Anderson, K. Doanay, and P. N. Pathirana, "Optimality analysis of sensor-target localization geometries," Automatica, vol. 46, no. 3, pp. 479-492, 2010.

[24] S. Zhao, B. M. Chen, and T. H. Lee, "Optimal placement of bearing-only sensors for target localization," in Proc. of 2012 American Control Conference, 2012, pp. 5108-5113.

[25] F. Zhang, "Geometric cooperative control of particle formations," IEEE Transactions on Automatic Control, vol. 55, no. 3, pp. 800-803, 2010.

[26] F. Zhang and N. E. Leonard, "Cooperative control and filtering for cooperative exploration," IEEE Transactions on Automatic Control, vol. 55, no. 3, pp. 650-663, 2010.

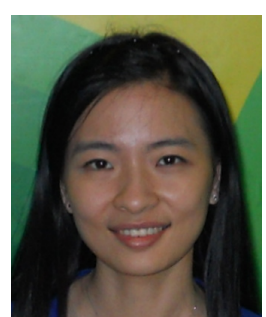

Wencen Wu received her B.S. and M.S. degrees from the School of Electronic, Information and Electrical Engineering at Shanghai Jiao Tong University, Shanghai, China, in 2006 and 2009, respectively, and her M.S. degree from the School of Electrical and Computer Engineering at the Georgia Institute of Technology, Atlanta, USA, in 2010, where she is currently pursuing a Ph.D. degree. Her research interests include bio-inspired autonomy, cooperative control, and multirobot exploration.

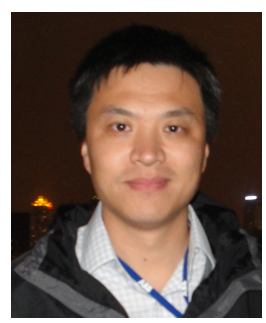

Fumin Zhang received the B.S. and M.S. degrees from Tsinghua University, Beijing, China, in 1995 and 1998, respectively, and the Ph.D. degree from the Department of Electrical and Computer Engineering, University of Maryland, College Park, in 2004. He has been an Assistant Professor in the School of ECE, Georgia Institute of Technology since 2007. He was a Lecturer and Postdoctoral Research Associate in the Mechanical and Aerospace Engineering Department, Princeton University from 2004 to 2007. His research interests include marine autonomy, mobile sensor networks, and theoretical foundations for battery supported cyber-physical systems. He received the NSF CAREER Award in 2009, and the ONR YIP Award in 2010. 